$\begin{array}{cl}\begin{array}{c}\text { Revue } \\ \text { de lhistoire } \\ \text { des religions }\end{array} & \text { Revue de l'histoire des religions } \\ & \begin{array}{c}\text { Figures de Noé de Gilgamesh au Coran } \\ \end{array}\end{array}$

\title{
La figure de Noé chez Philon d'Alexandrie et Flavius Josèphe
}

The figure of Noah in the works of Philo of Alexandria and Flavius Josephus

\section{Serge Bardet}

\section{(2) OpenEdition}

1 Journals

Édition électronique

URL : http://journals.openedition.org/rhr/8468

DOI : $10.4000 /$ rhr.8468

ISSN : 2105-2573

Éditeur

Armand Colin

\section{Édition imprimée}

Date de publication : 1 décembre 2015

Pagination : $545-565$

ISBN : 978-2-200-93012-7

ISSN : 0035-1423

Référence électronique

Serge Bardet, "La figure de Noé chez Philon d'Alexandrie et Flavius Josèphe », Revue de l'histoire des religions [En ligne], 4 | 2015, mis en ligne le 01 décembre 2018, consulté le 22 avril 2019. URL : http:// journals.openedition.org/rhr/8468; DOI : 10.4000/rhr.8468 


\section{La figure de Noé chez Philon d'Alexandrie et Flavius Josèphe}

Philon d'Alexandrie et Flavius Josèphe, deux juifs du $\mathrm{I}^{e r}$ siècle, partagent largement la même culture composite, mais leur traitement littéraire de Noé est extrêmement différent. Alors que Josèphe livre une paraphrase narrative, un récit d'un seul tenant, centré sur l'épisode du Déluge, Philon procède par allusions éparses, de type midrashique, dans des traités exégétiques et se concentre sur les suites du Déluge. De même, alors que Philon campe en Noé l'exemple de l'homme sage et juste, tantôt imparfait, tantôt exceptionnel, mais qui reçoit entièrement de Dieu sa sagesse, sans se faire enseignant et diffuseur de cette sagesse, Josèphe en fait une sorte de figure presbytérale, qui refonde les actes de piété, à travers le sacrifice et les règles de pureté.

\section{The figure of Noah in the works of Philo of Alexandria and Flavius Josephus}

Philo of Alexandria and Flavius Josephus, both Jews in the $I^{\text {st }} c$. CE, largely share the same composite culture; nevertheless, they deal very differently with Noah's character. While Josephus makes a narrative paraphrase, in one single and Flood-centric story, Philo refers to him through midrashic interpretations in many exegetical treaties and is focused on what he becomes after the Flood. Similarly, while Philo portrays an exemplarily wise and righteous man, sometimes imperfect, sometimes exceptional, but who only receives wisdom from God, without becoming a teacher of wisdom, Josephus makes him into a sort of priestly figure, who refounds acts of piety and rules of purity. 
Je suis l'esprit, vivant au sein des choses mortes. Je sais forger les clefs quand on ferme les portes ;

[...] Je m'appelle Bacchus, Noé, Deucalion.

Victor Hugo, La Légende des siècles ( $2^{\mathrm{e}}$ série),

Abîme - L'homme.

Deux principaux points communs justifient la comparaison du Noé de Josèphe avec celui de Philon : les deux auteurs sont deux juifs quasi contemporains et largement de même culture; tous deux font peser la même insistance sur la double figure du sage et du juste ${ }^{1}$. Mais on peut d'ores et déjà annoncer trois ou quatre oppositions, qui seront notre fil conducteur : opposition dans les choix narratifs ; dans la nature du commentaire ; opposition selon un axe être $v s$ agir ; opposition dans la relation de Noé à la question du Salut.

\section{DEUX CHOIX D’ÉPISODES RADICALEMENT DIFFÉRENTS}

Chez Josèphe, le personnage ne s'écarte guère de celui qui a été fixé par la Bible : il est lié au Déluge (Antiquités juives, I § 73-108, 140-142 pour l'essentiel). Chez Philon, en dehors des Quaestiones in Genesin, qui sont un commentaire linéaire du texte biblique (selon le genre des zètémata, des problèmes/questions et réponses), il semble bien n'y avoir que deux allusions au récit du déluge et à la navigation de Noé. Encore la première (De Migr. Abr., 125) est-elle très indirecte, à travers l'image du sage qui manque se noyer, mais surnage à grand-peine sur le flot, ce qui ne répond pas vraiment au texte biblique (on pense plutôt à un anti-Jonas). La seconde (De pramiis, 23), moins voilée, mais tout aussi implicite se fait par l'assimilation de Noé à Deucalion. Curieusement, dans son De vita Mosis ${ }^{2}$, Philon, qui évoque clairement le Déluge, évite soigneusement de nommer Noé : il recourt à des périphrases comme

1. Voir, par exemple, Philon, Leg. alleg., 77 [x2] ; Quod deterius, 121 ; Posteritate Caini, 48 ; Josèphe, Antiquités juives (désormais : AJ), I 75, 99. Doublet redondant chez Philon : il est juste et sage (De Abrahamo 27).

2. II 59-65. 
« une seule maison » ("̌v $\alpha$ oĩkov) ou «l'ancien et le chef de cette

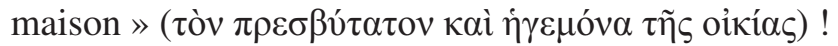

Pour le reste, on trouve une seule allusion au discours que Dieu tient à Noé avant le Déluge (Quod Deus..., 70). Partout ailleurs, dans une bonne trentaine d'occurrences, il est seulement celui qui s'enivre (Leg. alleg. 60-62), ou bien celui qui apprend la viticulture et, plus largement, l'agriculture.

\section{UNE PARAPHRASE NARRATIVE}

\section{CONTRE UNE EXÉGÈSE DE TYPE MIDRASHIQUE}

Josèphe en parle une fois pour toutes, sur le mode de la narration. Et il est très soucieux d'ancrer son personnage dans l'historicité (93-95 et 105-108). Pour cela, il recourt à trois moyens : la multiplication des «autorités » historiographiques ${ }^{3}$, souvent tombées dans l'oubli de nos jours (Bérose, Nicolas de Damas, Hiéronymos l'Égyptien, Mnaséas, Manéthon, Mokhos, Hestiée, « et beaucoup d'autres... ») ; l'attestation par des vestiges matériels (93-95; il ne saurait satisfaire à l'exigence d'autopsie : il ne les a pas vus lui-même et ne cache pas le caractère très indirect et hypothétique de sa connaissance, mais sa franchise et la répétition des attestations peuvent passer pour des garanties); le raisonnement visant à démontrer la plausibilité des faits rapportés (105-108).

Au contraire, Philon revient sur lui dans pas moins de seize traités différents, consacrés pour l'essentiel à l'interprétation allégorique. Cela, en tout cas, permet de prendre une première mesure de l'importance de Noé dans la démarche allégorique de Philon, puisque notre petit tour d'horizon va concerner douze de ses dix-sept traités allégoriques connus, soit deux tiers ${ }^{4}$.

On notera que la petiha, l'exégèse par la mise en relation d'un verset biblique avec un autre passage (généralement sans aucun rapport rationnel entre eux) est à peu près étrangère à l'un comme à l'autre : contrairement aux nombreux passages de TB Sanhedrin

3. Voir aussi Louis H. Feldman (2003), p. 403.

4. Pour un bref panorama introductif à ces apparitions de Noé, on aura paradoxalement intérêt à commencer par la conclusion de Jacques Cazeaux (1989), p. 265-269, intitulée « la disparition de Noé »! 
108 et de Genèse Rabba 30 auxquels le présent article va renvoyer, ni l'un ni l'autre de nos auteurs ne renverra à Job ou aux Proverbes pour comprendre la Genèse $e^{5}$. Philon utilise donc toujours le mode de l'exégèse midrashique et/ou philosophique (encore une fois, à l'exception des Quaestiones, qui relèvent des zètémata).

Maurice-Ruben Hayoun cite en plusieurs lieux une formule de Roger Le Déaut selon laquelle « le midrash se décrit, mais ne se définit pas ». Disons néanmoins que, par midrash, nous n'entendrons pas précisément ici « une exégèse autorisée de la Bible » (je souligne), mais certainement un commentaire herméneutique de l'Écriture, « la quête d'un sens profond, [voire] théologique », la mise au jour d'un «polysémantisme » au service d'une interprétation allégorique, au besoin par le biais «d'une philologie créatrice » (ici, c'est l'auteur qui souligne ${ }^{6}$ ).

Au cours de ces traités, Noé n'est pas du tout analysé de manière aléatoire et opportuniste, au hasard des associations d'idées ou de symbolismes faciles : conformément à la méthode allégorique si fréquente chez Philon, il apparaît plus comme un « leitmotiv» que comme un «motif». En d'autres termes, il est incorporé à l'architecture même de plusieurs traités ou cycles de traités. Il est inutile de répéter ici les études de Jacques Cazeaux ${ }^{7}$. Je me contenterai d'un ou deux ajouts.

Ainsi le De agricultura, le De plantatione, le De ebrietate et le De sobrietate constituent un cycle de quatre traités consacrés au même passage de Gn 9.20-21, les versets de l'ivresse de Noé. Philon ouvre le De agr. par deux paragraphes sur Noé comme viticulteur/agriculteur, avant de s'interroger sur le sens exact de l'expression « Noé le juste ». Par la suite, Noé est utilisé pour marquer le plan du traité : la formule « Noé fut cultivateur » réapparaît au § 20, c'est-à-dire après la fin de l'introduction et de l'exposé programmatique, à l'amorce de la première moitié de la dissertation sur la tâche des justes, qui sera soutenue par deux métaphores : le berger vs l'engraisseur de bétail et le cavalier vs l'homme qui monte sans savoir l'art hippique; au

5. Cf. La Gemara. Le Talmud de Babylone. Sanhédrin, t. 4, Paris, Colbo, 1973, introduction.

6. Pour tout ce paragraphe, cf. Maurice-Ruben Hayoun, La Littérature rabbinique, p. 20-22.

7. En particulier sa magnifique étude du Quod deterius, §119-140: Jacques Cazeaux (1983), p. 15-21. 
$\S 125$, pour marquer le début de la seconde moitié du traité, centrée sur le verbe n̋ $\rho \xi \alpha \tau o$ ([Noé] commença, se mit [à être cultivateur]) et consacrée au thème de la perfection, de la sagesse et des degrés de l'imperfection; puis (quatrième occurrence) embraye sur la conclusion au $\S 181^{8}$. La même citation ouvre ensuite le De plant. Et Noé est de nouveau évoqué au $\S 4$ du De ebr. Le personnage de Noé a donc bien une fonction d'ouverture, ou de réouverture, de chaque traité, des grandes étapes du cycle. Enfin, le De sobr., le dernier des quatre traités du cycle, ouvre de nouveau sur le personnage de Noé à travers le verset Gn 9.24, le retour à la sobriété initiale, et finit par un long développement visant à expliquer pourquoi c'est Canaan qui est maudit, alors que c'est Cham qui a vu la nudité de son père, passage encadré par deux évocations de Noé $(32,44)$. Entretemps, comme le fait remarquer Jean Pouilloux, l'éditeur du De plant., le renversement entre l'action du sage (à laquelle est consacré le De agric.) et les méfaits de l'aveuglement (auxquels est consacré le De ébr.), se fait au cœur du traité intermédiaire, le De plant., exactement au § 139: est-ce un hasard si le paradoxe de Noé, le sage qui s'enivre, réapparaît au $§ 140$, en ouverture à cette seconde moitié du cycle ?

Il y a donc bien là un élément architectural qui, non seulement scande le texte, mais introduit un élément de symétrie à l'intérieur du cycle des quatre traités qu'on peut ainsi qualifier de noachiques. De manière moins complexe, la répétition du verset Gn 6.8 (« Noé trouva grâce aux yeux du Seigneur ») sert de repère structurel dans le traité Quod Deus sit immutabilis : on remarquera en effet que les évocations de Noé et les citations de Gn 6.8 correspondent exactement aux débuts des cinq dernières parties du traité, sur les neuf que délimite A. Mosès dans l'introduction, p. 59-61; et que la septième partie (soit la partie médiane parmi ces cinq) est structurée par ces évocations/citations en son début (\$104), en son milieu (\$109) et en sa fin (\$116-117). Avant cela, les quatre premières parties du traité sont consacrées au repentir et à la colère de Dieu, et l'apparition de Noé marque le passage de la colère à la clémence divine.

On peut aussi remarquer que la mention de Noé ouvre la conclusion de la première partie du traité De migratione Abrahami (§ 125). Pour autant, Philon ne donne pas toujours l'impression d'écrire avec une

8. Je ne suis donc pas exactement le plan exposé par Jean Pouilloux dans l'introduction, p. 11. 
pleine cohérence d'une évocation à l'autre : tantôt il exalte en Noé le juste qui tend vers la perfection (Gig 1-5), tantôt il ne voit en lui qu'un juste incomplet, certes plus juste que ses contemporains, mais moins que les justes suivants $^{9}$ (De agricultura, De posteriate Caini 173-174). Le choix semble relatif au contexte : dans le De gig., Noé est opposé à ses contemporains pécheurs; dans les deux autres traités, il est mis en perspective avec le dépassement que constitueront Abraham et Moïse.

Dans un passage arithmologique d'inspiration probablement pseudopythagoricienne, peut-être néoplatonicienne ${ }^{10}$ (De posteritate Caini, 73), Philon explique que Noé est le nombre 10, parce qu'il est de la dixième génération de l'humanité ; il est en cela l'aboutissement de la première décade générationnelle, génération supérieure aux générations précédentes (dont Philon ne rappelle pas qu'elles ont été détruites par le Déluge, lequel reste obstinément dans l'implicite), mais encore inférieure à la décade suivante, qui part de Sem, le fils qui combat par le bien agir l'égarement de l'âme paternelle, pour aboutir à Abraham, modèle de sagesse sublime (§174), avec lequel se manifestent les premiers signes de l'émergence du peuple juif et de son alliance avec Dieu. Il est, selon le traité De congr. 90 (qui reprend en substance le même raisonnement), «le premier homme proclamé juste par les saintes Écritures », parce qu'il est celui qui «inverse le neuf » (les neuf générations humaines), «nombre superlativement ennemi », en dix, nombre de la perfection. C'est au point que, commentant le verset de Gn 6.9, qui entame la généalogie de Noé, Philon (De Abrahamo, 31; Quaestiones, I 97) comprend de la formule « Noé, homme juste, etc. » que la vertu lui tient lieu d'origine, d'ancêtre premier ${ }^{11}$, effaçant les neuf premières générations.

9. Idée reprise avec encore plus de sévérité dans TB Sanhédrin 108a, au nom de R. Yohanan : dans d'autres générations, il n'aurait pas mérité ce titre ; reprise et inversée par Rech Laqish : il a été juste dans une génération injuste, combien plus l'aurait-il été dans un meilleur contexte ! Tous deux sont des amoraim palestiniens de la première génération (début $\mathrm{III}^{\mathrm{e}} \mathrm{s}$.). Même discussion dans Genèse (Bereshit) Rabba, 30.9-10, mais au nom de R. Yehouda (b. Ilai) et R. Nehemia, deux tannaim de la quatrième génération (environ sous le règne d'Antonin le Pieux). Sur Noé le juste dans l'œuvre de Philon, voir l'exposé de Albert C. Geljon, p. 183-186 et, p. 191, le rapprochement entre Noé et le personnage du stage stoïcien.

10. Cf. fgt 11, attribué à Philolaos de Crotone (d'authenticité douteuse, cité dans Stobée, Eclogae, I 16.20), probablement dû à un lecteur du Timée ou à un héritier de l'Académie (Carl Huffman, p. 347-350); et Aristote, Métaphysique 986a8-12.

11. Attention comparable à ce curieux verset dans Genèse (Bereshit) Rabba, 30.5 . 
Chez Josèphe (\$152), les mêmes données ne sont pas du tout utilisées de la même manière : on retrouve la mention des dix générations, qui est facilement déductible du texte biblique, et la même mention d'Abraham comme repère de la vingtième génération. Mais cela ne sert à Josèphe qu'à établir une chronologie. La concordance des repères laisse penser que des réflexions làdessus circulaient assez largement dans les milieux cultivés ; mais le profit qu'ils en tirent l'un et l'autre est radicalement étranger.

Quoi qu'il en soit, le Noé de Philon n'est pas réellement un aboutissement, mais un intermédiaire entre la science (de Seth) et la sagesse (d'Abraham ${ }^{12}$ ). De même, il n'est pas aussi sage que Moïse $^{13}$ (Quod deus sit, 109). Il devient néanmoins dans le même traité, §122, 140, et dans le De gig. «le juste parfait», dont la justice fait d'autant plus ressortir, par contraste, l'injustice de ses contemporains, qui a provoqué le Déluge. Sa perfection se traduit et se prouve par le fait qu'il n'a eu que des fils, à l'exception de toute fille (ibid. §5).

\section{UNE FIGURE PLUTôt DANS L'ÊTRE, L'AUTRE PLUTÔT DANS L'AGIR}

Chez Philon, Noé ivre est une métaphore de l'âme passagèrement

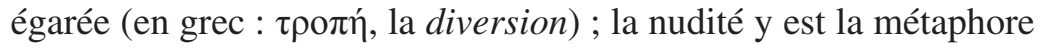
de l'égarement. Si cela reste de l'ordre du privé, dans le secret de l'âme, ce n'est pas très grave : la métaphore de l'ivresse dit aussi que l'homme sage et vertueux, une fois dégrisé, reprend le chemin et l'attitude de la sagesse (chez le méchant, ce n'est pas une ivresse, dont on se déprend bientôt, c'est une nature permanente). Mais si quelque chose en devient public, alors se produit de l'irréparable : Noé devient l'homme de la vertu perdue (ou regagnée, comme on le verra infra). Son égarement n'est pas par nature public : il s'est enivré « dans sa maison ${ }^{14}$ », ce qui aurait dû rester entre Dieu et lui, comme un égarement passager (Philon, comme la traduction des Septante, écrit $\dot{\varepsilon} v \tau \tilde{\varphi}$ oľk $\omega$, « dans sa maison », là où le texte hébraïque porte , «תוך הלהא , dans sa tente» : simple effet d'acculturation du texte). L'épisode est rendu public par son fils Cham, père de Canaan :

12. Cf. l'analyse complémentaire de Harry Wolfson, II, p. 182-183.

13. Voir Albert C. Geljon, p. 186sq.

14. §60, citation de Gn 9.21. 
chez Philon comme chez Josèphe ( $\$ 141)$, Cham est celui qui agit malignement en se riant de son père ${ }^{15}$ (ce que ne dit pourtant pas le texte biblique : il y a probablement un fait d'interprétation courante au I ${ }^{\text {er }}$ siècle), tandis que les deux autres fils, Sem et Japhet, couvrent sa nudité. À eux trois, ils symbolisent l'action de toutes les forces extérieures (personnes, actes, pensées...) qui agissent pour ou contre l'âme, pour sa perdition ou sa préservation ${ }^{16}$.

Là se situe un pont aux ânes de l'exégèse : pourquoi Noé maudit-il Canaan, fils de Cham, au lieu de maudire directement celui qui a révélé sa nudité ? Comment Noé le juste peut-il s'en prendre à celui qu'une morale immédiate (et, manifestement, dès l'Antiquité) tend à voir comme un innocent injustement pris pour cible ? La question se pose d'autant plus que la Bible n'apporte pas même un début d'explication ${ }^{17}$. Il y a manifestement des discussions actives làdessus, puisque Philon et Josèphe (comme plus tard les rédacteurs talmudiques) se sentent obligés d'apporter une explication, qui n'est pas pareille de l'un à l'autre.

Comme assez souvent, Josèphe $(\$ 142)$ est plus proche des traditions qui nous sont conservées par la littérature talmudique. Noé ayant déjà béni ses fils, ne pouvait plus maudire Cham et s'est donc reporté sur Canaan, un des petits-fils, ce qui a, en fin de compte, réduit le mal en évitant de faire porter la malédiction sur les autres fils de Cham ${ }^{18}$.

Philon développe une très longue explication (De sobr. 32-48), qui renvoie l'anecdote humaine de Gn 9.25 au monde des apparences et fait apparaître par l'étymologie une morale naturelle, on pourrait dire cosmique : il en ressort que Noé, Cham et Canaan sont des allégories. Le repos (ou plus exactement la permanence de l'être dans les attributs de son état : $\tilde{\varepsilon} \xi 1 \varsigma^{19}$ ) est une manifestation de la perfection.

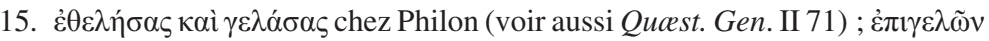
chez Josèphe. I 88.

16. Ou, dans le cas de Japhet, qui symbolisent l'indifférence : cf. Quast. Gen.,

17. Philon, Quasst. Gen., I 94 et II 9, puis TB Sanhédrin 108a étendent l'interrogation aux animaux, victimes du Déluge.

18. Même explication (entre autres hypothèses) dans Genèse (Bereshit) Rabba, 36.7 .

19. Il semble bien que Philon fasse référence à l'acception aristotélicienne (et non platonicienne) du terme : cf. Aristote Catégories, vIII, 9a10-13 ; cf. Otfried Höffe, p. 252-253. 
Or le nom de Cham signifie la chaleur ${ }^{20}$, qui est la condition, comme le parent, de l'agitation, laquelle est signifiée par le nom de Canaan,

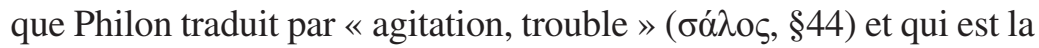
manifestation de l'imperfection, donc du mal.

Cette dernière traduction est moins évidente que pour le nom de Cham et renvoie probablement à la préposition $\supset$ (comme) et à la racine נוע (no'a, verbe signifiant tout ce qui est contraire à l'immobilité et à la stabilité : frémir, trembloter, onduler, ondoyer, tituber ${ }^{21}$, se troubler...), peut-être à la forme pilpel (intensive), נעה (nom dérivé l'agitation, le tourment, voire l'ivresse ; et נענע, adjectif : mouvant, mobile). On ne peut pas exclure que, par un jeu ironique, ce verbe fasse également quasi-homophonie avec le nom même de Noé (ก), glosé comme repos ${ }^{22}$.

Quoi qu'il en soit, Cham est donc «le mal en repos », tandis que Canaan est le mal en action. Or, on ne punit pas le mal qui reste virtuel, on punit seulement l'homme qui entreprend de passer à l'acte. Donc, malgré les apparences, la malédiction de Noé est bien conforme à l'ordre de la justice.

Ce sont ces malédictions qui, parce qu'elles se réalisent, permettent à Philon d'avancer que Noé le juste est une sorte de prophète : la forme interrogative qu'il y met (Quis rerum divinarum heres sit, 260) est largement rhétorique. Mais Noé le Juste s'oppose aussi à Caïn jusqu'à devenir plus ou moins une allégorie du libre $\operatorname{arbitre}^{23}$ (Quod deterius..., 122). À ce titre, plus que le juste, il est

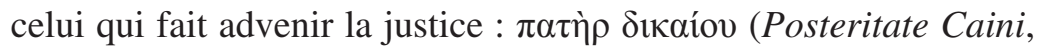
48); ou du moins qui le tente.

Chez Josèphe, Noé est, implicitement, celui qui a le souci de rétablir les règles de pureté. Il se trouve directement acteur dans ce que Gn 6.2 décrit plutôt comme un contexte : voyant que «de nombreux anges de Dieu s'unissaient à des femmes ${ }^{24} »$, il tente de

20. Même orthographe, חם: Philon joue donc de la quasi-homophonie en hébreu entre le nom de Cham et le terme qui désigne la chaleur (seul le vocalisme change).

21. cf. Ps, 107 (106). 27.

22. Legum alleg. III 77 et De Abrahamo, 27 ; racine identique נח, cf. Genèse [Bereshit] Rabba, 30.5. J'adresse mes remerciements à M. Vartejanu-Joubert pour ces indications.

23. Cf. Harry Wolfson, I, p. 451-452 à propos de Legum Allegoria, III 77.

24. Josèphe, tout comme Philon (De gig. 6 ; Quaestiones, I 92) fait partie de ceux qui interprètent comme anges les mystérieux Bnei-haÉlohim (בני-האלהים) 
les convaincre 25 de «changer leurs pratiques». Pourquoi est-il (comme le rédacteur biblique, mais plus explicitement encore) « scandalisé de leurs pratiques », si ce n'est parce qu'elles viennent en violation de prescriptions condamnant toute forme d'unions hétérogènes, toute hybridation ${ }^{26}$, prescriptions qui ne sont évidemment pas encore consignées dans Lv 19.19 au moment des faits, mais qui n'en sont, de ce fait, que mieux inscrites dans l'ordre du monde voulu par son créateur? Car Josèphe ajoute à l'anecdote quelque chose qui n'appartient pas au texte biblique : de cette union

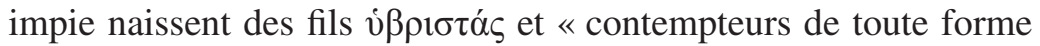

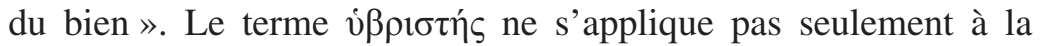
démesure tragique ${ }^{27}$. Il qualifie les prétendants de Pénélope, qui foulent aux pieds les lois morales ; les centaures, êtres hybrides par excellence; les forces brutes et dépourvues de conscience de la nature : éléments, animaux... Le texte biblique se contentait de voir en eux «les puissants (ou les forts : גברים) d'autrefois, hommes de renom ». Il y a bien une torsion du texte vers un propos normatif. Josèphe et son Noé auraient pu souscrire aux interdits renouvelés du traité Kilayim sur les unions hybrides ${ }^{28}$. D'une certaine manière, ils témoignent aussi d'un stade ancien de l'idée rabbinique selon laquelle la torah existait déjà avant la torah ${ }^{29}$.

du texte biblique. Mais Philon ne les relie pas à Noé. Références complétées et brièvement commentées par Étienne Nodet, $A J$, I $73 \mathrm{ad}$ n. 3.

25. Ce Noé militant, quasi-missionnaire, de la morale, étranger à Philon, se retrouve dans TB Sanhédrin 108ab (trois occurrences).

26. Selon les meilleures traditions manuscrites (mss SPLE : cf. É. Nodet, $A J$,

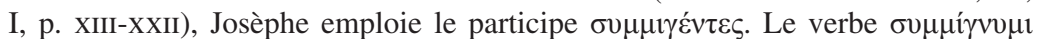
exprime souvent le mélange de deux matières distinctes, parfois l'union sexuelle ; mais, même dans ce dernier cas, cette union implique fréquemment des êtres différents (dieux et femmes dans l'Hymne à Aphrodite, 50) ou une idée de souillure, de déchéance (Euripide, Suppliantes, 222, 224).

27. Pace Louis H. Feldman (2003), p. 406sq.

28. Le lien entre Gn 6.12 et la question des hybrides est explicitement établi par Genèse (Bereshit) Rabba, 28.8 (interprétation rapportée aux noms de R. Yehouda bar R. Simon [= ha-Nassi] et de R. Itshaq, Tanna de la quatrième génération [milieu du $\mathrm{II}^{\mathrm{e}} \mathrm{s}$.]) ainsi que par TB Sanhédrin 108ab. Cf. Tos. Kilayim [Les Choses de nature différente] 5.5 (p. 222 éd. Liebermann) : une mule n'est ni cheval, ni âne, mais une espèce à part; M. Kilayim 8.4 , où on distingue selon le type de croisement. Voir aussi la question du fils mamzer dans TB Hulin (Les Animaux profanes) 78b-79a ; TJ Qiddusin [Les Fiançailles] 64c, 68b ; TB Yebamot [les Belles-sœurs, sur les règles du lévirat] 23a, 45a; Tos. Qiddusin IV.16 (baraita attribuée à R. Sim'on bar Yohaï, qui fleurit au milieu du $+\mathrm{II}^{\mathrm{e}} \mathrm{s}$.).

29. Discussion sur la même idée, concernant Philon, in Lewis, p. 55 (à propos des patriarches en général). 
Chez Philon, la fonction de «père de la justice» passe par la métaphore du cultivateur (Quod deterius, 105). «Noé fut cultivateur» : ainsi s'ouvre le traité De agr. D'un point de vue allégorique, qu'est-ce à dire? Si l'ivresse ou la sobriété sont les figures de la vertu et de l'égarement moral, la viticulture en particulier, l'agriculture en général sont une métaphore de la culture de l'âme, des soins qui lui sont apportés comme à une plante qu'il faut fortifier et faire fructifier; d'où cette expression tout à fait frappante selon laquelle l'éducation, la paideia, est « une géorgique

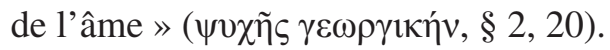

Mais cette métaphore est grosse de paradoxes : dans la Genèse, l'agriculture n'existait pas au sein de l'Éden (2.4-5) et c'est Caïn qui inventa l'agriculture (4.2). Elle n'est donc pas en elle-même une invention porteuse de bien. Philon doit donc amener son lecteur à clairement distinguer : Caïn n'a inventé que le travail de la terre

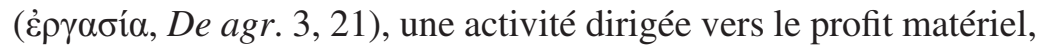
qui peut se passer d'un réel savoir agronomique et qui, plus encore,

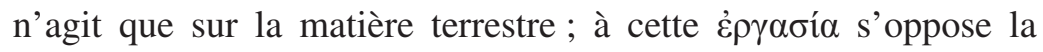
dignité d'une $\tau \dot{\varepsilon} \chi v \eta$; et, pour appuyer son raisonnement, Philon fait

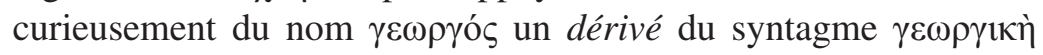
$\tau \varepsilon \dot{\chi} \chi \eta$ (alors que, linguistiquement, c'est évidemment l'inverse) : l'état d'agriculteur dérive de la préexistence d'une technè, tout comme l'apprentissage de la vertu et de la justice dérive de ce qu'il existe une vertu et une justice (ce n'est pas la vertu qui est semée dans les âmes par des travailleurs improvisés de la question ${ }^{30}$ ).

Malheureusement pour lui, Noé n'en est pas exactement à l'état de cultivateur : le texte philonien stipule que Noé se mit (ou commença) à

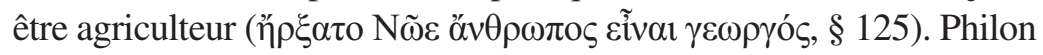
l'interprète alors à la lumière d'un dicton traditionnel selon lequel « le commencement ( $\alpha \rho \chi \eta ́$, le nom dont dérive le verbe á $\rho \chi 0 \mu \alpha$, ici sous la forme $\eta^{\prime} \rho \xi \alpha \tau$ ), c'est la moitié du tout » : Noé n'était donc qu'un

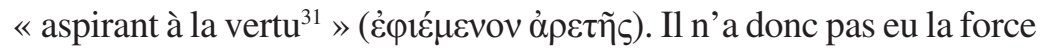
d'aller jusqu'à la perfection et s'est laissé gagner par l'ivresse.

L'autre paradoxe, c'est celui qui est pointé dans le De plant. 140-141 : si le cultivateur est la métaphore de celui qui cultive la

30. Cf. De Sobr. 38 : la vertu est un composé de prédispositions naturelles, de prescriptions légales et d'efforts constants.

31. Selon la traduction de Jean Pouilloux. 
justice et si le vigneron est par excellence le type de l'agriculteur (c'est le rappel du De agr. opéré au $§ 1$ du De plant. ${ }^{32}$ ), alors il s'ensuit que celui qui cultive la justice cultive dans le même mouvement la plante qui donne l'ivresse, métaphore du divertissement de l'âme dans l'erreur. Paradoxe apparent : en réalité, s'il y a toujours des pas grand-chose pour s'enivrer stupidement, le sage qui s'enivre reste sage. S'enivrer ( $\mu \varepsilon \theta v i \varepsilon v)$, c'est accomplir, parachever le sacrifice ${ }^{33}$ (* $\mu \varepsilon \tau \alpha-\theta v ́ \varepsilon v v$, faire les rites $\mu \varepsilon \tau \alpha \grave{\alpha} \theta v \sigma i ́ \alpha \varsigma)$, mais c'est aussi « relâcher l'âme » par l'ivresse ( $\mu \varepsilon \dot{\varepsilon} \theta \varepsilon \sigma ı \zeta<\mu \varepsilon \theta i ́ \eta \mu \mathrm{l} / / \mu \varepsilon \theta v ́ \varepsilon ı v)$ : relâchée, l'âme du sage répand un bonheur d'être sage comme celle de l'insensé se répand en sottises (De plant. 163-166).

Noé est donc à lui tout seul, chez Philon, tout un cheminement de sagesse. Au contraire, Josèphe souligne que les fils de Noé « tiennent leur abondance de la bienveillance divine ${ }^{34} »$, ce qui coupe court à toute spéculation sur le rôle et le sens de l'activité agricole.

\section{Chez Philon : Noé sauvé Par nature ou Par élection}

Dans le traité De somniis, 23, Philon détourne légèrement le sens

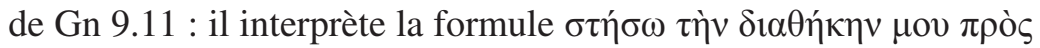
$\sigma \varepsilon ́$, non comme « J'établirai mon alliance avec vous », mais comme «Je ferai reposer sur toi mon alliance». Ainsi, «il gratifie cette

32. Au rebours de la spéculation philonienne, Genèse (Bereshit) Rabba, 36.3-4 fait de Gn 9.20 le début d'un itinéraire de déchéance : jeu phonique et sémantique sur ו-יחל (il commença), נתחלל (il déchut, se profana: verbe de même racine) et חולין (un être profane, homme ou animal). En effet, selon certaines traditions, la vigne est l'arbre de la faute d'Adam (il n'y a de fait aucune raison que ce soit un pommier avant la traduction latine de la Vulgate, qui joue sur l'homophonie mālum/mălum). Toujours selon ces traditions, il aurait mieux fait de planter un arbre plus « positif » : figuier, olivier... Finalement, il ne vaut pas mieux que Caïn et, commençant comme «homme juste » (Gn 6.9), il finit en «homme du sol» (am ha-arets, עם הארץ), accomplissant la courbe inverse de Moïse, d'abord qualifié «d'homme égyptien » (Ex 2.19) et finalement « homme d'Élohim» (Dt 33.1). Le rédacteur du midrash fait d'ailleurs remarquer que le texte biblique (Gn 9.21) n'est pas ויגלי (Noé (Noé se dénuda [de lui-même]), puis, par allitération, passe à l'idée d'exil (גלות) : Noé a été cause d'exil pour lui-même et pour les générations à venir. Sur Philon lui-même et sur la vigne de Sodome, voir les suggestions stimulantes de Jacques Cazeaux (1983), p. 107sq.

33. Ce qu'expose déjà Jacques Cazeaux (1989), p. 190sq.

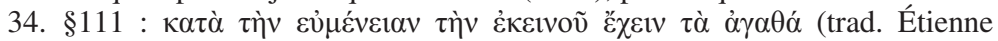
Nodet ; partout ailleurs, sauf précision contraire, nous proposons une traduction personnelle). 


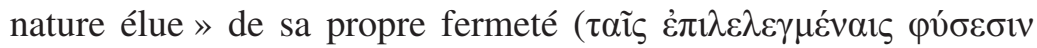

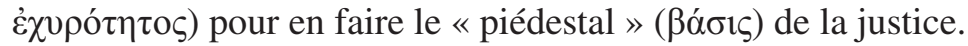

Peut-on alors penser que Noé, arrivé à ce degré de sagesse, et de culture de la sagesse, est devenu un maître instruisant les générations de la seconde humanité, comme le laisserait entendre l'expression «géorgique de l'âme », qui renvoie au genre éminemment hellénistique du traité technique, destiné à diffuser des savoirs ? De fait, dans un traité très marqué par le contexte hellénistique dans lequel il vivait (De promiis 23), Philon assimile explicitement Noé à Deucalion ${ }^{35}$, lequel s'inscrit pleinement dans le groupe de ces instituteurs de l'humanité sans lesquels le monde grec ne concevait pas les découvertes humaines.

Mais, pour le reste de l'humanité, la vraie source de vertu et de salut, ce n'est pas Noé, c'est Dieu : Noé n'est même pas l'agent de l'œuvre de justice en laquelle consiste l'alliance de Dieu avec son peuple; en donnant la justice aux hommes qu'il oblige, il ne

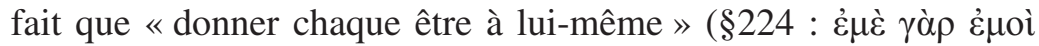

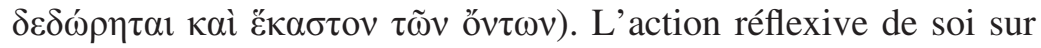
soi, le don réflexif de soi à soi fait de chacun, y compris Noé, un obligé de soi envers soi, car Dieu, qui a dispensé la sagesse, la justice, et la fermeté pour les faire fructifier, n'aurait que faire d'un échange de type évergétique ${ }^{36}$. Mais on comprend bien que, dans une relation ainsi exposée, il n'y a pas de place pour la prise en compte du magistère actif de moi vers autrui : je ne suis pas sauveur de mon prochain.

C'est ainsi que, dans le Quod deus sit, § 70, Noé est l'objet de la grâce divine (par opposition aux autres hommes, objets de sa colère). Ici, il n'est en rien salvateur, mais bien sauvé ${ }^{37}$. On peut aussi se reporter au $\S 86$, une discussion d'ordre juridique sur le verbe trouver et l'expression trouver grâce (< Gn 6.8; cf. aussi

35. Ainsi que le fait déjà remarquer É. Nodet dans sa n. 4, Josèphe, AJ, I 77 (et aussi $C$. Ap., I 130sq) fait implicitement l'assimilation de Noé à Deucalion, en

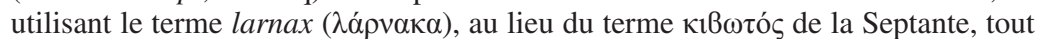
comme le font Plutarque (Mor. 968f) et Lucien (Syr. 12), dont le récit relatif à Deucalion est manifestement « normalisé » par le récit biblique. Il y a apparemment contamination croisée des deux traditions. Faut-il voir dans cette assimilation une façon, pour Philon, de convaincre son lecteur de l'historicité de Noé ? Pro Louis H. Feldman (2004), p. 86 ; contra Albert Geljon, p. 188.

36. Cf. Serge Bardet, p. 96-98 et 106.

37. Pace Annie Jaubert. 
$\S 104,116-117)$ : Noé trouve grâce ${ }^{38}$. Mais il reste celui qui «a

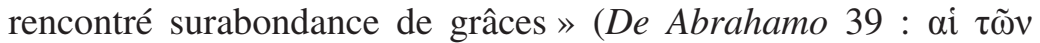

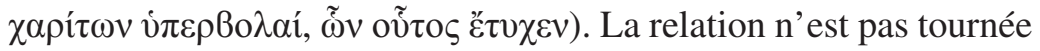
vers autrui.

Dans le traité De proemiis 22-23, où l'interprétation est on ne peut plus inspirée du contexte profane ou païen, grec et romain, dans lequel vivait l'Alexandrie de son temps, Philon fait de Noé, «le dernier de la génération condamnée et le premier de l'innocente ${ }^{39} »$, une sorte de champion de jeux sportifs ${ }^{40}$ : il est celui qui a une

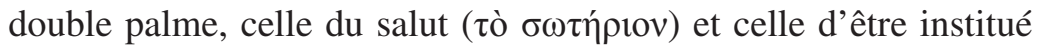

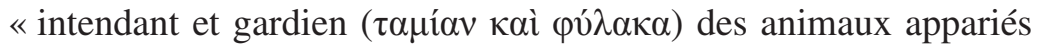

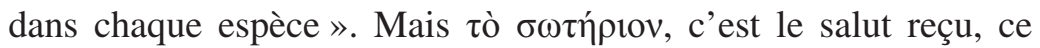
n'est pas l'action du sauveur (qui serait le féminin $\eta \dot{\gamma} \sigma \omega \tau \eta \rho i ́ \alpha)$. Être intendant et gardien des animaux, c'est, bien sûr, assurer leur salut quotidien (l'image du Bon Pasteur des évangiles n'est rien d'autre que cela à l'origine : imagerie hellénistique assez banale). Est-ce pour autant, ici, une métaphore d'une action salvifique auprès des hommes, comme le devient celle du Bon Pasteur ? Précisément, le texte se garde bien de toute allusion qui prêterait ou qui aiderait à une telle interprétation ${ }^{41}$.

Et, ainsi que Philon l'explique dans le traité De migr. 125-126, c'est en tant que sauvé ( $\left.\delta 1 \alpha \sigma \omega \theta \varepsilon \varepsilon^{4} \zeta^{42}\right)$ qu'il devient non plus vigneron ou agriculteur, mais lui-même arbre de vertu, étendant au loin ses racines, d'où naissent des surgeons vertueux, une humanité nouvelle dont Abraham, Isaac et Jacob ne sont pas les moindres

38. TB Sanhédrin 108a rapporte que, selon l'école de R. Yishmaël (tanna de la troisième génération, contemporain des empereurs Trajan et Hadrien), Noé avait d'abord été condamné, comme les autres hommes, puis finalement avait trouvé grâce. En effet, l'ordre du texte reflèterait la succession de la pensée divine. Cf. Genèse (Bereshit) Rabba, 28.9. Sans s'inscrire explicitement en faux contre une telle compréhension, les explications de Philon (\$104-108) tendent plutôt vers une conception inverse : Noé est le seul à ne pas avoir totalement trahi le don originel de la grâce à toutes les créatures et (Qucest. Gen., I 96) la grâce qui lui a été accordée est surtout d'avoir été à la fois le terme d'une humanité et l'origine d'une autre. Pour une approche en d'autres termes, cf. Jacques Cazeaux (1989), p. 96-98.

39. Traduction André Beckaert. De même pour la citation suivante.

40. Parallèlement se livre une véritable compétition vers toujours plus de mal chez ses contemporains : De Abrahamo, 40.

41. Du reste, Philon n'intègre pas Noé à la (brève) liste des plus grands pasteurs d'hommes dans le De agric., 42-43 et leur définition (\$ 41, 44-45) ne correspond guère à Noé.

42. Même verbe dans Josèphe, Contre Apion, I 130. 
représentants. Le salut qu'il peut apporter à sa famille et aux espèces animales est totalement absent du texte philonien.

\section{Chez JosèPhe : NoÉ SaUVÉ/SaUVeur de TyPe Presbytéral ?}

Le Noé de Josèphe est également sauvé $(\$ 76,78)$; mais beaucoup plus tourné vers autrui, que ce soit vers Dieu ou vers les hommes. Déjà, avant le déluge, il tente d'avertir ses contemporains (AJ, I 74). Il n'est d'ailleurs pas écouté. Ensuite, après le déluge, il a une dimension de piété active par le sacrifice offert à Dieu, dimension qui correspond au texte biblique (Gn 8.20). En cela, Noé s'oppose aux hommes de sa génération, qui « ne rendirent plus à Dieu les honneurs qui étaient de règle ». De son côté, Josèphe est en parfaite opposition à Philon, qui ne mentionne jamais (pour ce qui concerne Noé) cet aspect de la vertu et du respect des choses divines. D'autre part, il amplifie le motif par rapport au texte matrice, puisque son Noé procède aux sacrifices à pas moins de trois reprises : à peine débarqué, « il sacrifie à Dieu et fait un festin avec sa maisonnée » (\$92); ce sacrifice est immédiatement doublé de victimes ou

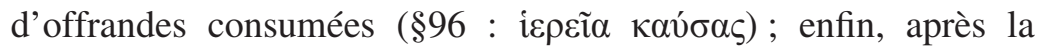
première vinification, il fait de nouveau un sacrifice et un banquet

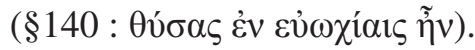

Il y a donc un décalage par rapport au texte biblique : seul le deuxième sacrifice correspond plus ou moins au texte biblique ; les deux autres sont une adjonction de Josèphe ${ }^{43}$, qui n'emploie aucun terme technique qui permettrait de déterminer à quel type de pratique sacrificielle il fait référence. Il ne fait aucune allusion aux règles de pureté des animaux sacrifiés.

Quoique Josèphe évite absolument tout terme technique du sacrifice, on peut, néanmoins, essayer de les caractériser : le premier (Josèphe utilise seulement le participe $\theta u ́ \sigma \alpha \varsigma$ ) est plutôt, ici, un sacrifice de paix (korban shelamim), de louange ou d'action de grâce, ou encore plus probablement, un sacrifice présenté au lecteur sur le mode gréco-romain de l'alliance verticale avec la divinité, puis du lien horizontal entre les hommes lors du festin. Dans le

43. Tout au contraire de Josèphe, Genèse (Bereshit) Rabba, 30.6 explique que, le lion ayant frappé et mutilé Noé au moment de sortir de l'arche, ce dernier n'était plus habilité à sacrifier; c'est donc Sem qui procède aux sacrifices, par la suite. 


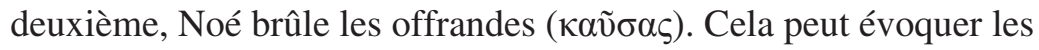
holocaustes du texte biblique ('oloth), mais rien n'indique que les offrandes aient été animales (et, de fait, l'ambiguïté remonte à la

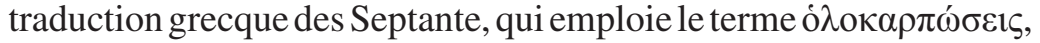
étymologiquement lié aux offrandes végétales, mais dont l'usage, en contexte scripturaire, s'attache aux holocaustes d'animaux ${ }^{44}$ ). Le troisième est assez clairement un sacrifice visant à faire agréer les prémices de la récolte par la divinité, ce qui est également très conforme aux pratiques gréco-romaines. Il y a donc chez Josèphe, tout aussi clairement, la volonté de camper Noé dans un personnage soit de prêtre ${ }^{45}$, soit de pater familias (c'est-à-dire, de toute façon, dans un rôle de prêtre du périmètre domestique ${ }^{46}$ ) : l'humanité étant réduite à la seule famille de Noé, la distinction entre culte public et culte domestique est à peu près impossible à établir. C'est peut-être en vertu de cette indistinction originelle que la part du sacrifice qui revient aux hommes (et par laquelle deux des trois sacrifices ne sont pas techniquement 'oloth [holocaustes], mais bien de l'ordre des korbanoth chelamim) n'est pas partagée entre cohanim (qui n'existent pas encore) et familiers, mais consommée par Noé et sa maisonnée indistinctement.

Quoi qu'il en soit de ce dernier point, il ne semble pas que

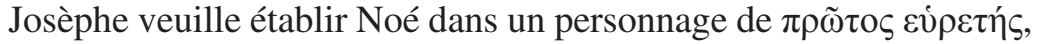
d'inventeur de telle ou telle fête, de tel ou tel rite. Le sacrifice offert à l'occasion des prémices est étranger, par exemple, à l'offrande des bikkourim, qu'il évoque superficiellement, mais qui ne concerne pas la vigne et requiert lévites, orphelins et veuves que, pour des raisons diverses, Noé serait bien en peine de trouver ${ }^{47}$ : il ne peut certainement pas passer pour une sorte d'institution primitive de cette fête. Aucun élément ne permet non plus de penser aux prescriptions sur l'offrande végétale en Lv 2. Aucun détail concret ne permet d'assimiler le

44. Dans la religion gréco-romaine, les deux peuvent être brûlés et i $\varepsilon \rho \varepsilon i ̃ \alpha$ est encore plus vague.

45. Josèphe aussi était prêtre : faut-il voir dans les §96-98 une mise en miroir de leurs épreuves, comme semble vouloir le suggérer très discrètement Michael Tuval, p. 179 ?

46. Cf. John Scheid, p. 121 : « le chef de famille fixait le calendrier, déterminait les divinités à honorer, et célébrait les sacrifices familiaux à l'intérieur de la maison et dans les lieux de culte publics. » Mutatis mutandis, c'est bien ce que fait le Noé de Josèphe.

47. Dt 16.9-12. 
premier sacrifice au korban pessah (le sacrifice pascal). Certes, Noé, « après avoir sacrifié, fit un festin avec sa maisonnée », ce qui peut faire penser au seder pascal ; malheureusement, il est probable que le rituel du seder s'est constitué au cours de l'époque romaine et Josèphe ne pourrait guère entreprendre de le faire remonter à Noé par une simple allusion elliptique, comme si son ancienneté était une chose entendue. D'ailleurs, l'absence de toute céréale cultivée à ce stade du récit rend un peu vain le parallèle avec cette fête éminemment agraire (et exclut Shavouot). Soukkot est certes liée à la vigne, comme le troisième sacrifice ; mais est-ce suffisant (ou alors le Noé de Josèphe s'exprimerait-il comme l'inconscient, par inversion et contradiction : il fait explicitement le deuxième sacrifice pour prévenir le retour du déluge, quand Soukkot comprend une dimension propitiatoire, pour s'assurer le retour des pluies) ? Et le sacrifice du milieu : si ce n'est celui de Shavouot, pourrait-ce être celui de Rosh ha-Shana ou de Kippour ? Aucun détail ne permet un tel rapprochement.

Bref, aucun des trois sacrifices n'est la préfiguration d'un sacrifice distinct, ultérieurement établi, ils sont l'acte même du sacrifice dans quelques modalités fondamentales et il semble que Josèphe vise plutôt à construire un personnage qui opère une transition aussi réussie que possible entre la première humanité et celle qui se reconstruit : d'une part il clôt efficacement le divorce initial entre Caïn et Abel, comme entre Caïn et Dieu, puisqu' il obtient l'agrément d'une offrande agricole après l'avoir, à une ou deux reprises, obtenu d'une offrande animale. D'autre part, il constitue, avant Loth, avant Abraham, avant Melchisédech, une sorte d'antétype presbytéral : pieux, juste, maître du rituel. Comme Loth, il est sauvé avec les siens parce qu'il fait exception à l'iniquité ambiante. Comme Abraham, il est auprès de Dieu un intercesseur efficace $(\$ 101)$. Comme Melchisédech, « roi de justice », il s'associe au vin (mais pas au pain : il n'a pas encore replanté de céréales, du moins pas dans l'économie du texte $\left.{ }^{48}\right)$. Et, comme dans la Bible, il devient (après Adam, mais pour la seconde humanité) le premier dépositaire des règles de pureté alimentaire $(\$ 102)$.

48. C'est pourquoi il est impossible d'interpréter le premier sacrifice plus précisément, comme un sacrifice de reconnaissance (korban toda), indissociable du pain. 
Et pourtant, curieusement, Josèphe esquive la formulation de l'alliance passée par Dieu avec les hommes ${ }^{49}:$ au $\S 103$, dans la phrase qui correspond presque exactement au verset de Gn 9.13, il remplace le terme d'alliance ou de pacte ( $\delta\llcorner\alpha \theta \dot{\kappa} \kappa \eta$ en grec de la Septante comme chez Philon) par celui de cessation ( $\pi \alpha \tilde{\nu} \lambda \alpha v$, terme très imprécis). Mais ce n'est pas propre à l'épisode de Noé : c'est une constante chez Josèphe ${ }^{50}$. Il est donc probable que cette esquive du thème de l'alliance, quelles qu'en soient les raisons, n'a pas de poids sur le sens qu'on doit donner à ce passage précisément.

On aura donc compris que Noé constitue une figure extraordinairement plastique : homme métaphore chez Philon, homme juste, avec ce que cette expression indique de qualités et de limites, figure du pieux sauvé, il est chez Josèphe une sorte d'antétype presbytéral « universel », c'est-à-dire accessible à la compréhension d'un lecteur gréco-romain.

L'idée que s'en font Josèphe et Philon a peu de rapports, a priori, avec la tradition véhiculée par les manuscrits qumrâniens. Il y a plus de proximité, dans les préoccupations exprimées ou sous-jacentes et dans les façons d'aborder le personnage, avec la littérature talmudique ; mais ils en ont une approche beaucoup plus laudative, beaucoup moins critique que celle des rabbins du Talmud. De fait, les Noé de Josèphe et de Philon campent des figures beaucoup plus chargées de valeurs symboliques, on pourrait dire théologiques, que dans les traités et midrashim postérieurs.

sbardet@univ-evry.fr

49. La chose peut sembler curieuse parce que, lorsque le terme d'alliance (ברית) apparaît dans la Bible, en Gn 6.18, la promesse en est précisément faite à Noé et que, ensuite (Gn 15.9-18), elle est préparée par un sacrifice.

50. Ainsi que le fait remarquer Étienne Nodet, $a d$ n. 8 du $§ 103$. 


\section{BIBLIOGRAPHIE}

\section{SOURCES}

La Gemara. Le Talmud de Babylone. Sanhédrin, t. 4, Paris, Colbo, 1973.

Midrach rabba, t. 1, Genèse rabba, traduit par Bernard MARUANI et Albert Cohen-Arazi ; annoté et introduit par Bernard Maruani, Paris, Verdier, 1987.

Talmud Yerusalmi, traité Qiddouschin, Paris, Maisonneuve et Larose, 1887.

\section{ARISTOTE}

Catégories, traduction de Jules Tricot, Paris, Vrin, 1959.

Métaphysique, t. 1, traduction de Jules Tricot, Paris, Vrin, 1991.

Flavius JosèPHe

Antiquités juives, traduit et annoté sous la direction d'Étienne NoDET, Paris, Le Cerf, 1992.

Contre Apion, traduit par Léon BLum, Paris, Les Belles Lettres, 1930.

Huffman (Carl A.) (éd.), Philolaus of Croton. Pythagorean and Presocratic, Cambridge U. P., 1993.

Philon D'AleXANDRIE

Arnaldez (Roger), Pouilloux (Jean), Mondésert (Claude) (éd.), Les Euvres de Philon d'Alexandrie :

2) Legum allegoriae, introduction, traduction et notes par Claude MondésERT, Paris, Le Cerf, 1962.

5) Quod deterius potiori insidiari soleat, introduction, traduction et notes par Irène FeuER, Paris, Le Cerf, 1965.

6) De posteritate Caini, introduction, traduction et notes par Roger Arnaldez, Paris, Le Cerf, 1972.

7-8) De Gigantibus. Quod Deus sit immutabilis, introduction, traduction et notes par André Mosès, Paris, Le Cerf, 1963.

9) De agricultura, introduction, traduction et notes par Jean Pouilloux, Paris, Le Cerf, 1961. 
10) De plantatione, introduction, traduction et notes par Jean PouIlloux, Paris, Le Cerf, 1963.

11-12) De ebrietate. De sobrietate, traduits par Jean Gorez, Paris, Le Cerf, 1962.

14) De migratione Abrahami, introduction, traduction et notes par Jean Cazeaux, s. j., Paris, Le Cerf, 1965.

16) De congressu, eruditionis gratia, introduction, traduction et notes par Monique Alexandre, Paris, Le Cerf, 1967.

19) De somniis, introduction, traduction et notes par Pierre SAVINEL, Paris, Le Cerf, 1962.

20) De Abrahamo, introduction, traduction et notes par Jean Gorez, Paris, Le Cerf, 1966.

27) De promiis et pænis. De exsecrationibus, introduction, traduction et notes par André BeckAERT, Paris, Le Cerf, 1961.

34) Quastiones in Genesim (livres I-II), introduction, traduction et notes par Charles Mercier, Paris, Le Cerf, 1979.

\section{ÉTUDES}

BARDET (Serge), « Flavius Josèphe et l'évergétisme : un regard juif sur un échange perverti », Kentron (Caen), 28, 2012, p. 89-110.

Cazeaux (Jacques), La trame et la chaîne, I : Les Structures littéraires et l'exégèse dans cinq des traités de Philon d'Alexandrie, Leyde, Brill, 1983 (Arbeiten zur Literatur und Geschichte des hellenistischen Judentums 15).

Cazeaux (Jacques), La trame et la chaîne, II : Le Cycle de Noé dans Philon d'Alexandrie, Leyde, Brill, 1989 (Arbeiten zur Literatur und Geschichte des hellenistischen Judentums 20).

Feldman (Louis H.), «Questions about the Great Flood, as Viewed by Philo, Pseudo-Philo, Josephus, and the Rabbis », Zeitschrift für die alttestamentliche Wissenschaft, 115, 2003, p. 401-422.

Feldman (Louis H.), “Remember Amalek!” : Vengeance, Zealotry, and Group Destruction in the Bible According to Philo, Pseudo-Philo, and Josephus, Cincinnati, Hebrew Union College Press, 2004.

Geljon (Albert C.), « Philo's interpretation of Noah », in Stone (Michael E.), Aminay (Aryeh) et Hillel (Vered), dir., Noah and his Book(s), Atlanta (Ga.), Society of Biblical Literature, 2010, p. 183-191.

Hayoun (Maurice-Ruben), La Littérature rabbinique, Paris, P.U.F. («Que sais-je ?» $\left.\mathrm{n}^{\circ} 2526\right), 1990$.

HöFFE (Otfried), Aristoteles-Lexikon, Stuttgart, Kröner, 2005. 
Jaubert (Annie), «Le Thème du "reste sauveur" chez Philon », in [Arnaldez (Roger), MondÉsert (Claude) et Pouilloux (Jean), edd.], Philon d'Alexandrie, Paris, CNRS, 1967, p. 243-253.

LEwIS (Jack P.), A Study of the Interpretation of Noah and the Flood in Jewish and Christian Literature, Leyde, Brill, 1968.

Mayer (Gunther), Index Philonicus, Berlin \& New York, De Gruyter, 1974.

Nikiprowetsky (Valentin), Le Commentaire de l'écriture chez Philon d'Alexandrie : son caractère et sa portée. Observations philologiques, Leyde, Brill, 1977.

Scheid (John), La Religion des Romains, Paris, Armand Colin, 2010 $\left(1998^{1}\right)$.

Tuval (Michael), « The Role of Noah and the Flood in Judean Antiquities and Against Apion by Flavius Josephus », in STone (Michael E.), Aminay (Aryeh) et Hillel (Vered), dir., Noah and his Book(s), Atlanta (Ga.), Society of Biblical Literature, 2010, p. 167-181.

Wolfson (Harry A.), Philo. Foundations of Religious Philosophy in Judaism, Christianity, and Islam (2 vol.), Cambridge (Mass.), Harvard U. P., 1947. 